\title{
Altered Expression of mRNA and miRNA during Mechanical Support of the Failing Human Heart
}

\author{
Marguérite E.I. Schipper' ${ }^{1}$, Sjoukje I. Lok ${ }^{2}$, Hub Dullens ${ }^{1}$, \\ Joyce Van Kuik¹, Frits H.J. Gmelig-Meyling', Jaap Lahpor'2, \\ Marc A. Vos 4 , Arnoud Van Der Laarse ${ }^{5}$, Nicolaas De Jonge², \\ Matthijs F.M. Van Oosterhout ${ }^{1}$ and Roel A. De Weger ${ }^{1}$ \\ ${ }^{1}$ Depts of Pathology, ${ }^{2}$ Heart and Lung, ${ }^{3}$ Immunology, and ${ }^{4}$ Medical Physiology, \\ University Medical Centre Utrecht, Utrecht \\ ${ }^{5}$ Dept of Cardiology, Leiden University Medical Center, Leiden \\ The Netherlands
}

\section{Introduction}

Remodeling during heart failure is characterized by structural rearrangement of the cardiac ventricular wall architecture. It involves hypertrophy of cardiomyocytes, fibroblast proliferation, and increased deposition of extracellular matrix (ECM) proteins (Brower et al., 2006). Support of the left ventricle with a Left Ventricular Assist Device (LVAD) in patients with end-stage heart failure results in less neurohormonal activation (Estrada-Quintero et al., 1995; Frazier and Myers, 1999; Bruggink et al., 2006a), improvement of the patient's general condition (De Jonge et al., 2001; Grady et al ., 2003), reduction in ventricular diameter (reverse remodeling), and limited recovery of contractile elements in cardiomyocytes (Muller et al., 1997; De Jonge et al., 2002). Furthermore, reduction of ECM volume (Milting et al., 2008; Goldsmith and Borg , 2002; Bruggink et al., 2006b), diminished production of tumor necrosis factor (Thohan et al., 2005; Bruggink et al., 2008), and reduction in brain natriuretic protein serum levels (Bruggink et al., 2006a; Kemperman et al., 2004) have been described during LVAD support. The changes in ECM during this process of reverse remodeling resulted not only in a time-dependent change of type I and type III collagen protein (Goldsmith and Borg, 2002; Stamenkovic, 2003), but also in considerable changes in composition of the basal membrane. These included amongst others reduced collagen type IV content in the cardiomyocyte basal membrane, as a result of increased matrix metalloproteinase activity (Bruggink et al., 2007; Spinale, 2002; Li et al., 2001; Klotz et al., 2005). So, during LVAD support myocardial architecture and composition change at the level of both the cardiomyocytes and the ECM.

The mechanics of the heart require a close interplay between cardiomyocytes and the ECM (Parker and Ingber, 2007) and therefore, one may anticipate a coordinated change in the molecules responsible for this interaction. These changes may not be the same in all heart failure patients supported by a mechanical support device. Some patients' hearts may improve and may be eligible for removal of the support device without a heart transplantation (bridge to recovery and weaning from the device), whereas others do not 
improve on support or may even deteriorate and these patients remain on the device (destination therapy) or will ultimately receive a heart transplant (bridge to transplantation). To make the proper choice of the type of therapy for each patient a good set of (bio)markers is required (De Weger and De Jonge, 2009).

In this chapter, we describe whether mRNA expression patterns could be indicative for the state of heart functionality supported by a LVAD (Heart-Mate I, Thoratec, Pleasanton, CA, USA). The changes in mRNA profiles that are detectable in myocardial biopsies taken from patients with end-stage heart failure due to dilated cardiomyopathy (DCM) or ischaemic heart disease (IHD) before and after LVAD support were analyzed, and compared with biopsies taken from control hearts as a reference (Table 1). Furthermore, the expression of 109 genes is described, which are involved in the process of mechanotransduction in the heart. Their expression was studied by Quantitative(Q)-PCR. The cohort comprised selected genes coding for ECM filaments (such as collagens), transmembrane proteins (molecules that connect cells and matrix components like integrins and sarcoglycans), intracellular molecules, adhesion molecules related to mechanotransduction and signal transduction, ion-channel molecules, and factors involved in pro- and anti-fibrotic processes. The expression of mRNA is not always directly related to protein production, due to posttranscriptional regulation. Recently, it is has been shown that intracellular gene expression is regulated in part by small RNA molecules: microRNAs (miRs). These miRs are highly expressed in heart tissue (Ji et al., 2007; Cheng et al., 2007) and have also been related to heart diseases (Chen, 2007; Van Rooij et al., 2006). The list of regulatory miRs involved in heart disease is constantly increasing (Coutinho et al., 2007; Markham and Hill, 2010). Each miR can regulate various mRNA expressions and which mRNA is

\begin{tabular}{llllll}
\hline Nr & Age & Diagnosis & Gender & Days on LVAD & Medication during LVAD-support \\
\hline & & & & & \\
1 & 56 & IHD & Male & 138 & None \\
2 & 57 & IHD & Male & 225 & None \\
3 & 45 & IHD & Male & 259 & $2,5 \mathrm{mg}$ Ramipril \\
4 & 57 & IHD & Male & 263 & None \\
5 & 36 & IHD & Male & 325 & $2 \times 4$ mg Perindopril \\
6 & 26 & IHD & Male & 357 & None \\
7 & 39 & IHD & Male & 548 & $3 \times 6,25$ mg Capoten \\
8 & 34 & DCM & Female & 55 & $3 \times 6,25$ mg Capoten \\
9 & 17 & DCM & Male & 111 & None \\
10 & 47 & DCM & Male & 190 & None \\
11 & 35 & DCM & Male & 196 & None \\
12 & 32 & DCM & Female & 219 & 3 mg Captopril \\
13 & 25 & DCM & Male & 263 & $1 \times 25$ mg Losartan \\
14 & 32 & DCM & Male & 286 & 4 mg Perindopril \\
15 & 25 & DCM & Male & 330 & $2 \times 10$ mg Fosinopril \\
16 & 46 & DCM & Male & 484 & $3 \times 50$ mg Capoten \\
\hline
\end{tabular}

DCM: dilated cardiomyopathy, IHD: ischemic heart disease, LVAD: left ventricular assist device.

Table 1. Patient characteristics. 
regulated by which $\mathrm{miR}$ is not determined with certainty for most genes (see www.targetscan.org; Schuldt, 2010).

An additional goal of this study was therefore, to analyse the changes in expression of 4 miRs that are known to be expressed in the myocardium (Chen, 2007; Ikeda et al., 2007): miR-1, miR-133a, miR-133b and miR-208. These miRs have been related to heart failure. The expression of these miRs was measured in the same group of patients used to analyse the mRNA expression after LVAD support. Our purpose was to find out whether the LVADinduced remodeling of the heart was accompanied by changes in the expression of miRs that could influence the protein expression of the mRNA studied. This could make expression of some mRNAs less suitable as biomarker for the assessment of the functional quality of the supported heart. If this is the case the expression of miRs may serve as better markers either in the myocardium or the serum.

\section{Tissue distribution of mRNA and miR in the myocardium}

Tissue samples taken form various locations in cross sections of the heart showed that the expression of both $\mathrm{miR}$ in the right and left ventricular wall did not show significant variation. Only in the infarcted area the expression of miR was low to absent. Similar data were obtained for mRNA (Figure 1).

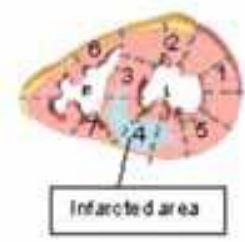

A

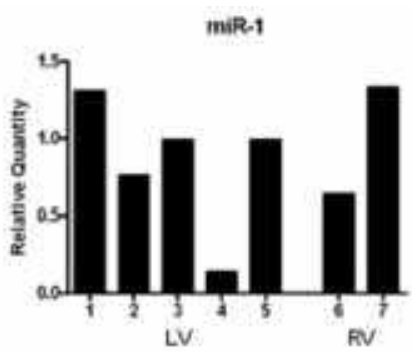

B

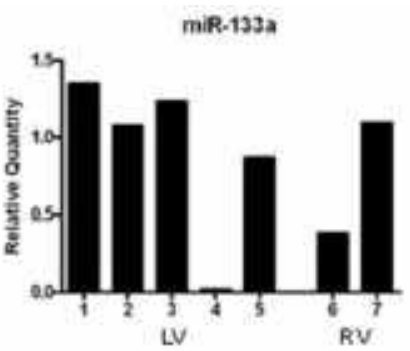

C

Fig. 1A. Circular cross section of the heart indicating the biopsy areas.

$\mathrm{B}$ and $\mathrm{C}$. miRNA expression in and around the infarcted area in the indicated biopsies of a representative case. The miRNA expression in an infarcted area (biopsy 4) is much lower than in the surrounding areas. LV and RV = left and right ventricle.

\section{Hierarchical clustering of gene expression in myocardial tissue of IHD and DCM patients}

The gene profiles in DCM and IHD heart tissue, detected by Q-PCR, were compared using TIGR software (www.tm4.org). Figure 2 shows the whole data set for all pre-LVAD samples versus the median of control samples. Hierarchical clustering was performed on all 92 detectable genes. The genes that were not detectable $(n=14)$ and house keeping genes $(n=3)$ were excluded (Table 2). The clustering segregated two groups: one group consists of 6 DCM and 1 IHD patients and the other group consists of 5 IHD patients with 2 DCM patients. One DCM and one IHD patient were clustered outside both groups. So, there is a strong tendency of segregation between IHD and DCM. Therefore, the DCM and IHD patient groups were analyzed separately in this study. 


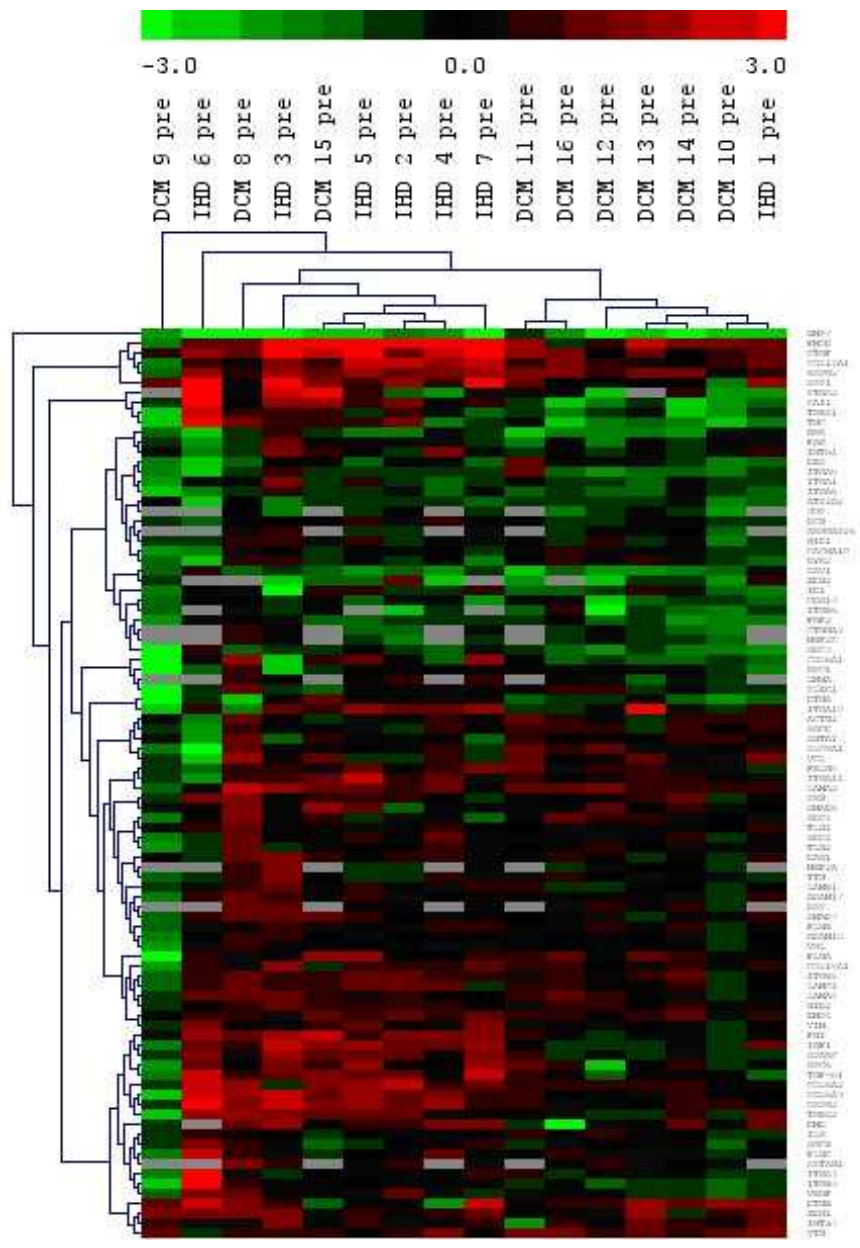

Fig. 2. Unsupervised hierachical clustering of myocardial gene expression profiles pre-LVAD in IHD and DCM patients. Clustering was performed on all 92 detectable genes. Unsupervised hierarchical clustering was performed on normalized data using the multiexperiment viewer (MeV, version 4.3) of the TIGR software (www.tm4.org). The Reletive quantity (RQ) of each sample per gene was normalized:

Normalized signal of sample $\mathrm{x}=\log 2$ (RQ sample $\mathrm{x} /$ median RQ).

To compare DCM and IHD the median was taken from the RQ (relative quality) of control hearts. To compare pre- and post-LVAD in DCM and IHD samples, the median of all DCM or all IHD samples were taken, respectively. Clustering was performed on the whole dataset, and distance metric selection (Euclidean distance) and linkage metric selection (Complete linkage clustering) were used (www.tm4.org). This segregated two groups; one group consisting of $6 \mathrm{DCM}$ patients with $1 \mathrm{IHD}$ patient, and the other of 5 IHD patients with 2 DCM patients. Two patients (one DCM and one IHD) clustered outside these groups. Red: mRNA expression is higher than the median of control hearts. Green: mRNA expression is lower than the median of control hearts. Grey: not done. 

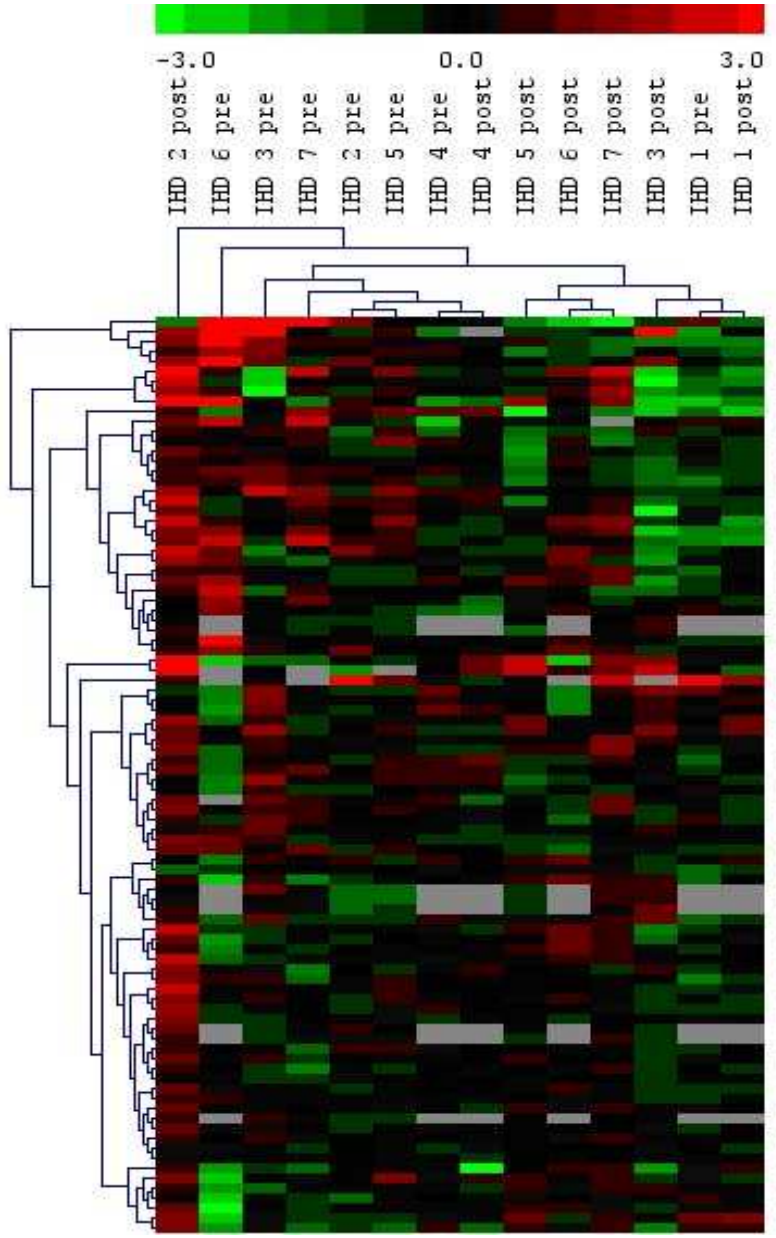

Fig. 3. Unsupervised hierarchical clustering of myocardial gene expression profiles pre- and post-LVAD in IHD patients.

Clustering was performed on all 92 detectable genes and it segregated the patient group into a pre- and post-LVAD group. See for explanation Figure 2. Red: mRNA expression is higher than the median of all IHD samples. Green: mRNA expression is lower than the median of all IHD samples. Grey: not done.

The expression of 14 genes was below level of detection and are therefore not included in this table: ADAM 12 (ADAM metallopeptidase domain 12), ADAM 15 (ADAM metallopeptidase domain 15), AGC1 (aggrecan 1), ANK1 (Ankyrin 1), DSPG3 (dermatan sulfate proteoglycan 3), EDN3 (endothelin 3), LAMC3 (laminin, gamma 3), MMP-7 (matrix metallopeptidase 7), MUC16 (mucin 16), NOS1 (nitric oxide synthase 1), SCN1A (sodium channel, voltage-gated, type I, alpha), SCN2A2 (sodium channel, voltage-gated, type II, alpha 2), SDC1 (syndecan 1), TNXB (tenascin XB). 


\begin{tabular}{|c|c|c|c|c|c|c|c|c|c|c|c|}
\hline \multirow[b]{2}{*}{$\begin{array}{l}\frac{2}{8} \\
8 \\
0\end{array}$} & \multirow[b]{2}{*}{$\begin{array}{l}\frac{\mathrm{E}}{\mathrm{E}} \\
\frac{\mathrm{E}}{\mathrm{c}} \\
\frac{\mathrm{c}}{\mathrm{E}} \\
\mathrm{g}\end{array}$} & \multirow[b]{2}{*}{$\begin{array}{l}\text { ga } \\
\vdots \\
\vdots \\
\vdots \\
\vdots\end{array}$} & \multirow[b]{2}{*}{ 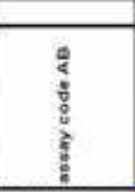 } & \multicolumn{4}{|l|}{ OCH } & \multicolumn{4}{|l|}{ MO } \\
\hline & & & & 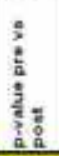 & 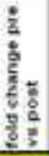 & 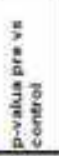 & 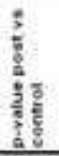 & 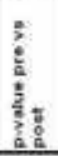 & 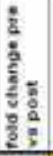 & 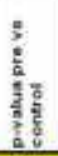 & 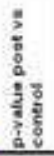 \\
\hline \multirow[t]{12}{*}{ ECM } & 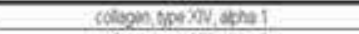 & Cक:4ती & 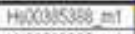 & 0 ons & 1,75 & $05: 5$ & 005 & 054 & & 0.014 & 0,011 \\
\hline & 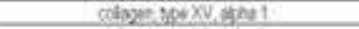 & CQHAI & Howeran $=1$ & 0.91 & 1,26 & 035 & 0151 & 002 & & 035 & 0.51 \\
\hline & 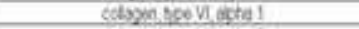 & Coleal & 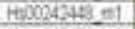 & 0.04 & & 0716 & 0181 & 0972 & & 8996 & 0451 \\
\hline & 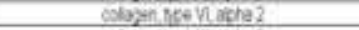 & $006+2$ & Hsom $42464=1$ & 0,13 & & 0,129 & 0.121 & 0,489 & & 0081 & 0,195 \\
\hline & cologyn toq VI ache 3 & Cos(4) & 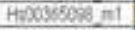 & 0,042 & 7,53 & 0.64 & 008 & Q701 & & 0.075 & 061 \\
\hline & 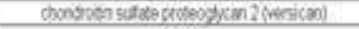 & $\operatorname{css} 2$ & $1+900171642=1$ & 0000 & & 040 & 034 & $007 \%$ & & 0,065 & 0472 \\
\hline & deche & DOI & Hoxostases =1 & 0,049 & 86 & $0 \cos 1$ & 080 & 0273 & & 0352 & 0924 \\
\hline & tains & FEOS & Hexp: & 0,43 & & 040 & 0,2 & 0,21 & & Q44! & 060 \\
\hline & throcoodan & $F M C O$ & HDotstig al & 0350 & & 0097 & 2186 & 0002 & 807 & 0,00 & 0.34 \\
\hline & Ftrocosten! & FN: & HWX0415000 iㅔ & 045 & & 0.59 & $0 \%$ & 0,004 & 076 & 0,03 & 0,59 \\
\hline & 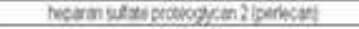 & HSPG! & HoDisation & 0004 & 80 & 0891 & 2007 & 0,805 & & 0052 & Q.122 \\
\hline & orvonedin. & SPATC & Homptrat & $05 \%$ & & 070 & 028. & 0.140. & & 0018. & 018 \\
\hline \multirow[t]{5}{*}{ AFF } & 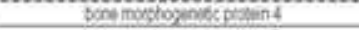 & Etou & 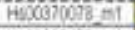 & 0009 & 0,21 & 0215 & 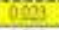 & 0,120 & & Dotit & 0064 \\
\hline & 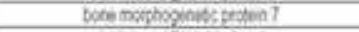 & EM? & Heopsesteg=1 & 0.044 & 2.24 & 0,008 & 0,32 & 0022 & & 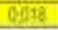 & 0,23 \\
\hline & 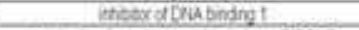 & Dt & 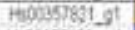 & 0.091 & 089 & 0.90 & 0.88 & Q32 & & Q.56 & 059 \\
\hline & 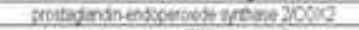 & PTES: & Hoot55139 int & 004 & & 0911 & 0351 & 0757 & & 0445 & 0406 \\
\hline & 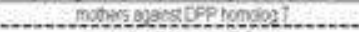 & SMPT & Hoostroos -1 & 0.300 & & 0522 & Q8E. & 026. & & $00 \mathrm{~s}$ & 863 \\
\hline \multirow{8}{*}{ pif } & 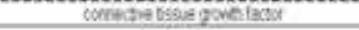 & CTE & 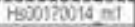 & 0258 & & 0.318 & 0002 & 0350 & & dots & 0,112 \\
\hline & ouselet & ECAI & Hoponzesolet & 0310 & & 0352 & 0262 & 0913 & & 0060 & 0,94 \\
\hline & insane? & $E C W 2$ & Homer5ib-1 & 044 & & 0,93 & 0115 & 0.419 & & 0267 & 0,130 \\
\hline & Sholastgow forter? & EEF 2 & H:00086645 $\times 1$ & 0,300 & & 002 & 0369 & 043 & & 0,113 & 085 \\
\hline & 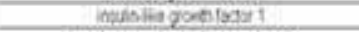 & SGE! & $1000153126=1$ & 0006 & & 0838 & 014 & 0.003 & & 0003 & Dore \\
\hline & mosm oyankDPP tambog 6 & SMOD & He0076579 01 & 0023 & & 0,53 & 0.881 & 0125 & & 6511 & 0000 \\
\hline & Indionng gountass beth 1 & $T+5-4$ & Hopol:137 $=1$ & 0,555 & & 0,728 & $0,0 \%$ & 0221 & & 0311 & 08655 \\
\hline & 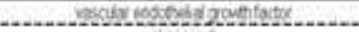 & JEE. & Haceses. & 085. & & 083. & 014. & 024. & & 082. & $05 \mathrm{P}$ \\
\hline \multirow[t]{17}{*}{$8 \mathrm{M}$} & gricant 1 & से & A- $00151805=1$ & 0,545 & & 0.738 & 0.960 & 025 & & 0330 & OTA \\
\hline & $\sec \cos 6$ & $\operatorname{CPC} 5$ & Heptivent $\mathrm{El}$ & 0200 & & $10 \overline{0}$ & 0973 & 002 & & 600 & 0,0 \\
\hline & Iming eshnt2 & Low2? & HEDO160308 $=1$ & $O, F, 3$ & & 0.072 & 0111 & $67 \mathrm{~m}$ & & 0028 & 8002 \\
\hline & invingospot & 12424 & 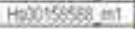 & 005 & & 025 & $0(35)$ & 023 & & 0610 & 295 \\
\hline & Gain, bete 1 & LASE1 & Hepols6030 $=1$ & 0001 & 682 & 0368 & 0.35 & $0 \times 6$ & & 068 & 0201 \\
\hline & 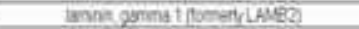 & LAMC1 & Hocosctuso $=1$ & 0058 & og & 0273 & 6994 & 0238 & & 0028 & 0009 \\
\hline & $\operatorname{imn} A E$ & Wha & HOPMTSUR2 $=1$ & 0.351 & & 0227 & 0.717 & 0,938 & & 0.055 & 0.112 \\
\hline & niosyent 1 & 100 & HeDtsoled & 0,24 & & 0434 & 0973 & 0.783 & & 0.971 & 0953 \\
\hline & 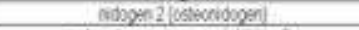 & 102 & $H=0201293=1$ & $88 \pi$ & & 0.850 & 0851 & 0336 & & 0798 & 0332 \\
\hline & 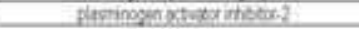 & PA! & 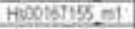 & 0.91 & & 034 & $0+\sqrt{6}$. & 0,34 & & 049 & 067 \\
\hline & Perdin 1 & PECI & He0056977-1 & 0,45 & & 0.053 & 0110 & Q6151 & & 05,4 & 0234 \\
\hline & sposond & socd & 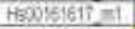 & 0,45 & & 0596 & 0.803 & 0,242 & & 0.49 & 0805 \\
\hline & cetwosorion & SPPI & 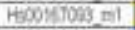 & 0,001 & .127 & $0 \times 5$ & 0022 & 609 & 1. Ala & 0.04 & 0.155 \\
\hline & trimborpeognt & Desi & $\mathrm{H}_{5001702 \mathrm{~S}} \mathrm{mt}$ & 0.93 & & 0,04 & 048 & 0.45 & & Q81 & 0 t32 \\
\hline & trintocepogen? & THE: & $1600570248=1$ & 068 & & 0.785 & 0.523 & 0,364 & & 0040 & $0 \times 4$ \\
\hline & teranone & WN & Hompes & 0.24 & & 039 & c5s & 009 & & 0539 & $0 \leqslant 4$ \\
\hline & xaxoes & Vis & Henogoes $=1$ & 904 & & 00010 & 0081. & 0171. & & 0.368 & 2071 \\
\hline TaM & 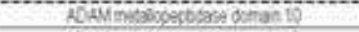 & ACokistio & $1+60013563=1$ & 0965 & & 0699 & 0544 & 6358 & & 685 & $62 \pi$ \\
\hline & AOdMmat Woscossesa doman it & ADLMIT: & Hexo23422: $=1$ & 0251 & & 0.83 & 0224 & $05 ;$ & & 0051 & 0,095 \\
\hline & 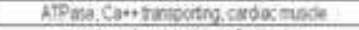 & APVA? & $\mathrm{H}=00135929 \mathrm{a}$ & 0.496 & & $0.0 \%$ & 0.109 & ogke & To & $0 \mathrm{~m}$ & 0.21 \\
\hline & 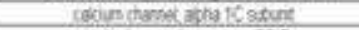 & SAONA1C & 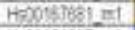 & 0,098 & 0.82 & 0206 & 038 & 004 & & $0, x_{3}^{3}$ & 0,89 \\
\hline & 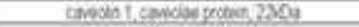 & Chit & $1+20146992-1$ & 026 & & 0011 & os: & 0442 & & 0,51 & 01,4 \\
\hline & cogeth $13, \mathrm{HCog}$ en & (conti) & 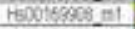 & 803 & 80 & Q.118 & 0350 & 0.05 & Bत!! & 0,568 & 02814 \\
\hline & ontoyian 1 & Caत1 & 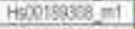 & 0,324 & & 0.59 & 0128 & 0,009 & & 0.244 & 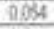 \\
\hline & Espin sota! & BaA1 & Hes00236030 m1 & 804 & 298 & 004 & 0500 & 0294 & & 0988 & 059 \\
\hline & Iftegrin acta to & ronos & HoDortur23, ni & 0023 & 247 & 0651 & 0000 & 6332 & & G017 & 0072 \\
\hline & Itign ofta it & FG:1 & Heoos/927_mit & 0,30 & & 0264 & (0): & 0581 & & 0258 & $\sqrt{0043}$ \\
\hline & irtogn athe! & MGAS & $1+0 m 9372=1$ & 099 & & 0856 & 0525 & 0304 & & 0339 & 0,818 \\
\hline & eggrn, attas & Fot5 & $H: 00: 33732=1$ & $03 \%$ & & 0206 & $0 \% 5$ & 009 & 041 & 0810 & 03867 \\
\hline & Astateng & TUR3 & $1-00173052=1$ & 0001 & 1,9 & 0025 & 0457 & 0060 & 1.23 & 0249 & 0303 \\
\hline & tarsospeat & RGAT & 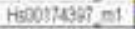 & 0002 & 007 & $0 . \overline{2}$ & 0515 & 0401 & & 0237 & 0350 \\
\hline & menin Des! & ECB1 & Hsuossos:5s al & 0239 & & 0,313 & COBS & $03 \pi$ & & 0747 & $08 \%$ \\
\hline & $a_{6} 2 \operatorname{mon}_{0} 683$ & 903 & Hexpr73978 el & 0.08 & & $06+3$ & 0578 & 032 & & 0466 & $0 \pi 00$ \\
\hline & nepin Deas & Cues & Hexo600606 $=1$ & 0.570 & & 0232 & 0.132 & 0,410 & & 0012 & 035 \\
\hline & negrabes 6 & 8665 & $H_{3000}=168458=1$ & 0,163 & & 0,38 & 6602 & 0008 & 93 & Q0t0 & 020 \\
\hline & propocin 3 & $50 c^{3}$ & Hemoconget & 0002 & 19 & 0006 & $0+62$ & 6885 & & 0345 & 0,25 \\
\hline & incogican bets & 8608 & 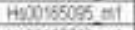 & 0107 & & 0030 & 005 & $00 \mathrm{di}$ & 078 & 0303 & 0,000 \\
\hline & saccogycs dset & SGCO & H:00155726 $\mathrm{ml}$ & 0598 & & 0.153 & 0031 & 0058 & 10 & 6382 & 0,002 \\
\hline & 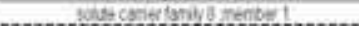 & scest & Hoorsus2.8t & 0589 & & 0282. & 0285. & 0810. & & 050 & 0.180 \\
\hline
\end{tabular}




\begin{tabular}{|c|c|c|c|c|c|c|c|c|c|c|c|}
\hline \multirow[b]{2}{*}{ 迹 } & \multirow[b]{2}{*}{ 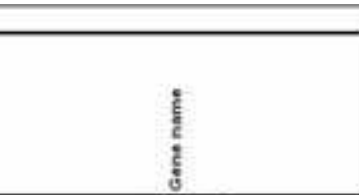 } & \multirow[b]{2}{*}{ 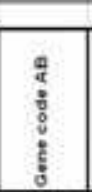 } & \multirow[b]{2}{*}{ 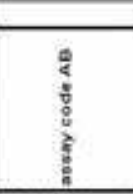 } & \multicolumn{4}{|l|}{ OCM } & \multicolumn{4}{|l|}{ MD } \\
\hline & & & & 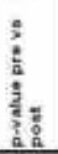 & 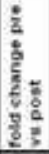 & 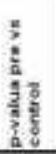 & 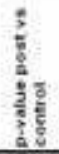 & 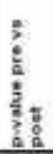 & 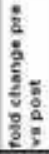 & 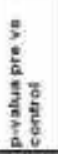 & 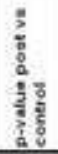 \\
\hline \multirow[t]{19}{*}{$F$} & 30 ain aghat? & ACTR2 & Hopors500 =1 & 0,055 & & 0213 & 0.569 & $0.17 \pi$ & & 0.910 & 0.972 \\
\hline & 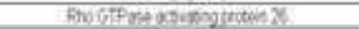 & AfHSapy & 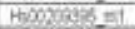 & 024 & & 0,80 & $62 x$ & 8,30 & & $0 \times 6$ & 045 \\
\hline & onsmit & DES & $100057258=1$ & 0496 & & 0,617 & 0158 & 082 & & 0,59 & 0.13 \\
\hline & grstoshes & $D N D^{\circ}$ & Hoonothes of & 0,106 & & 02008 & 0.203 & 0852 & & 000 & 0,04 \\
\hline & Onnefian & OSP & Hexoteg422 $m 1$ & 0,559 & & $0 \% 9$ & 0367 & 0,015 & InI & 02818 & 0,120 \\
\hline & ontroonith arte & ong & H.2008601 & 0.428 & & 0335 & 0673 & 0800 & & 0894 & 0835 \\
\hline & Gsacreva beta & On 8 & H50022285 of & 0.488 & & D04 & 0004 & 0313 & & 0,163 & .023 \\
\hline & timina whe & FIM & How0155065 =1 & $0, m i$ & & 0.331 & 006 & 0,575 & & 0.155 & 024 \\
\hline & Amne bets & FUE & 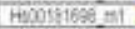 & 0003 & 892 & 0835 & 0034 & Qim & & 0,151 & 0008 \\
\hline & Elancingernga & ANE & Hososs5:24 mi & 0078 & & 0248 & 6854 & 6,765 & & 6677 & 0604 \\
\hline & posangowoden & LP & $1+00159400$ - & 0855 & & 0050 & 000 & 0.94 & & 0,03 & 000 \\
\hline & perstn & Fxy & $1+2 \times 00004=1$ & $00 \pi$ & & 0544 & 012 & 0,301 & & 642 & 0215 \\
\hline & 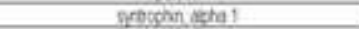 & 9त्ती & HWox:015 o1 & 0312 & & (iii) & 105 & 0.454 & & 0,972 & $0 \% 2$ \\
\hline & spectinaper & SPTAUI & $\mathrm{H}=00062200^{\circ}=1$ & 0.048 & & 0861 & 0353 & 0005 & 056 & 0848 & 0232 \\
\hline & 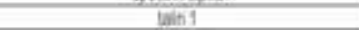 & TUN & Hoxos:075 of & 0,053 & & 02250 & $0.2 \pi$ & 0370 & & 0.19 & 000 \\
\hline & $\tan 2$ & $T M R$ & $\mathrm{H} 500522257 \mathrm{~m}$ & 0005 & 111 & 0295 & 6022 & 0215 & & 0428 & 0.30 \\
\hline & $m$ & $\pi N$ & H5) $0096225 \mathrm{~m}$ & 0,300 & & 0803 & 0201 & 0,5 & & 0951 & 0.195 \\
\hline & vecin & SC & 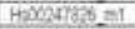 & $0 \operatorname{mg}$ & & Q15A & $0 \sin$ & $00 \mathrm{~m}$ & & 0.04 & $010:$ \\
\hline & vithet & VM & 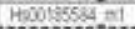 & 0304 & & 08283 & 0.53 & $0555^{2}$ & & 0.350 & 044 \\
\hline \multirow{9}{*}{ sif } & 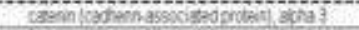 & chomis & How32805281 & $03 \pi^{2}$ & & 6036 & 6800 & 60002 & 190 & 6.353 & 60432 \\
\hline & 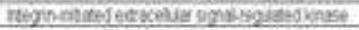 & EDK & 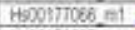 & 0,12 & & 0,050 & 088 & 0,435 & & 0435 & 0,838 \\
\hline & 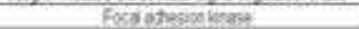 & $F A K$ & $1+2007828 \mathrm{mi}$ & 0,00 & PSP & 0,312 & $64 \alpha$ & Q275 & & 097 & 0,91 \\
\hline & 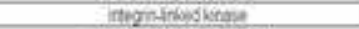 & IK & $1+2017794=1$ & $05 \times 6$ & & $05 \%$ & 0,33 & 0.97 & & 062 & 0130 \\
\hline & nocges ensoxcerfade $2 \mathrm{~A}$ & IE $2 A$ & Hoc0271535 of & 0.164 & & 0232 & 0.664 & 0,030 & 689 & 0359 & 0,005 \\
\hline & 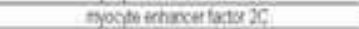 & $167 x$ & Hoxesthe $\mathrm{mt}$ & 0 क्ष & & 0564 & 0.200 & 0,002 & De & 030 & 0.886 \\
\hline & 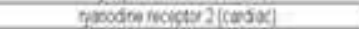 & F:P2 & Hopostusi & 0.045 & 2,20 & 0476 & 0007 & 0,03 & iो & 038 & 000 \\
\hline & spoecint. & 501. & $1+0,1755=1$ & 0345. & & 0828 & 0.85 & 100 & & 089. & 092 \\
\hline & 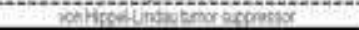 & He & 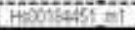 & 024 & & 636 & $6 \%$ & कीक & & 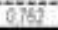 & 6034 \\
\hline
\end{tabular}

Table 2. Statistical analysis of gene expression profiles in DCM and IHD patients. Significant changes are indicated in yellow. The genes are grouped by function/location. Abbreviations: extracellular matrix proteins (ECM), pro- and anti-fibrotic factors (P/AFF), basal membrane proteins (BM), transmembrane and adhesion molecules (TAM), intracellular filaments (IF), and signal transduction factors (STF). Applied Biosystems (AB). $>$ and $<$ indicate whether gene expression is significantly higher or lower compared to control.

\section{Hierarchical clustering of gene expression in myocardial tissue pre- and post-LVAD support}

Hierarchical clustering of the IHD samples showed a clear segregation into a pre- and a post-LVAD group (Figure 3). In DCM patients a similar segregation into a pre- and a postLVAD group was not evident (data not shown).

\section{Differential expression of genes in myocardial tissue pre- and post LVAD}

Changes in gene expression were tested individually using the paired t-test in DCM and IHD separately. Furthermore, these gene profiles were compared with gene profiles of controls to test whether gene profiles normalized or showed a tendency to deviate from normal after LVAD therapy using the unpaired t-test. Table 2 shows all genes investigated, grouped by function/ location: extracellular matrix proteins (ECM), basal membrane proteins (BM), transmembrane and adhesion molecules (TAM), intracellular filaments (IF), 
signal transduction factors (STF) and pro- and anti-fibrotic factors (P/AFF) with the pvalues and fold changes.

In Table 3 only the genes that show significant changes are indicated for DCM and IHD patients separately. Only a minority of genes showed a significant difference between preand post-LVAD: DCM 19/92 genes (21 \%) and IHD 12/92 genes (13\%). Most of these genes showed an upregulation post-LVAD (DCM 18/19 genes and IHD 8/12 genes). In DCM preLVAD 6 genes and post-LVAD 9 genes were upregulated compared to control. Only one gene, encoding caveolin, showed a decreased expression in both pre- and post-LVAD compared to control. In IHD pre-LVAD 12 genes were upregulated and 2 downregulated compared to control. Post-LVAD 6 were upregulated and 2 downregulated. Among these, two genes (dystrophin and laminin gamma 1) showed an increased expression compared to control in both pre- and post-LVAD samples.

DCM

\begin{tabular}{|c|c|c|c|}
\hline 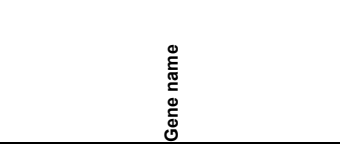 & 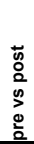 & 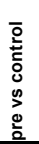 & $\begin{array}{l}\overline{0} \\
\overline{0} \\
0 \\
0 \\
0 \\
0 \\
0 \\
0 \\
0\end{array}$ \\
\hline osteopontin & $\boldsymbol{\nabla}$ & $=$ & $<$ \\
\hline bone morphogenetic protein 4 & $\Delta$ & $=$ & $>$ \\
\hline collagen, type $\mathrm{VI}$, alpha 3 & $\mathbf{\Delta}$ & $=$ & $>$ \\
\hline filamin B, beta & $\Delta$ & $=$ & $>$ \\
\hline laminin, gamma 1 & $\Delta$ & $=$ & $>$ \\
\hline ryanodine receptor 2 (cardiac) & $\Delta$ & $=$ & $>$ \\
\hline talin 2 & $\Delta$ & $=$ & $>$ \\
\hline cadherin $13, \mathrm{H}$-cadherin (heart) & $\mathbf{\Delta}$ & $=$ & $=$ \\
\hline $\begin{array}{l}\text { calcium channel, alpha } 1 \mathrm{C} \text { subunit } \\
\end{array}$ & $\mathbf{\Delta}$ & $=$ & $=$ \\
\hline collagen, type XIV, alpha 1 & $\mathbf{\Delta}$ & $=$ & $=$ \\
\hline collagen, type $\mathrm{XV}$, alpha 1 & $\mathbf{\Delta}$ & $=$ & $=$ \\
\hline decorin & $\mathbf{\Delta}$ & $=$ & $=$ \\
\hline focal adhesion kinase & $\mathbf{\Delta}$ & $=$ & $=$ \\
\hline heparan sulfate proteoglycan 2 (perlecan) & $\mathbf{\Delta}$ & $=$ & $=$ \\
\hline inhibitor of DNA binding 1 & $\mathbf{\Delta}$ & $=$ & $=$ \\
\hline integrin, alpha 1 & $\Delta$ & $=$ & $=$ \\
\hline integrin, alpha 10 & $\Delta$ & $=$ & $=$ \\
\hline laminin, beta 1 & $\mathbf{\Delta}$ & $=$ & $=$ \\
\hline bone morphogenetic protein 7 & $\mathbf{\Delta}$ & $<$ & $=$ \\
\hline integrin, alpha 6 & $\boldsymbol{\Delta}$ & $<$ & $=$ \\
\hline dystrobrevin beta & $=$ & $>$ & $=$ \\
\hline vitronectin & $=$ & $>$ & $=$ \\
\hline integrin, alpha 11 & $=$ & $=$ & $>$ \\
\hline sarcoglycan, beta & $=$ & $=$ & $<$ \\
\hline fibroblast growth factor 2 & $=$ & $<$ & $=$ \\
\hline junction plakoglobin & $=$ & $<$ & $=$ \\
\hline caveolin 1 & $=$ & $<$ & $<$ \\
\hline
\end{tabular}

IHD

\begin{tabular}{|c|c|c|c|}
\hline 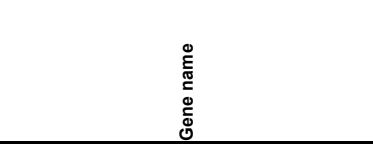 & 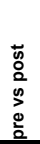 & 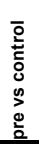 & $\begin{array}{l}\overline{0} \\
\overline{2} \\
0 \\
0 \\
0 \\
0 \\
0 \\
0 \\
0\end{array}$ \\
\hline fibronectin 1 & $\mathbf{\nabla}$ & $>$ & $=$ \\
\hline integrin, alpha 5 & $\boldsymbol{\nabla}$ & $=$ & $=$ \\
\hline osteopontin & $\nabla$ & $=$ & $=$ \\
\hline sarcoglycan, beta & $\boldsymbol{\nabla}$ & $=$ & $=$ \\
\hline ryanodine receptor 2 (cardiac) & $\Delta$ & $=$ & $>$ \\
\hline cadherin $13, \mathrm{H}$-cadherin (heart) & $\Delta$ & $=$ & $=$ \\
\hline calcium channel, alpha $1 \mathrm{C}$ subunit & $\Delta$ & $=$ & $=$ \\
\hline catenin (cadherin-associated protein), alpha 3 & $\Delta$ & $=$ & $=$ \\
\hline desmoplakin & $\Delta$ & $=$ & $=$ \\
\hline integrin, alpha 6 & $\Delta$ & $=$ & $=$ \\
\hline integrin, beta 6 & $\mathbf{\Delta}$ & $=$ & $=$ \\
\hline myocyte enhancer factor $2 \mathrm{~A}$ & $\Delta$ & $=$ & $=$ \\
\hline myocyte enhancer factor $2 \mathrm{C}$ & $\Delta$ & $=$ & $=$ \\
\hline spectrin alpha & $\Delta$ & $=$ & $=$ \\
\hline ATPase, Ca++ transporting, cardiac muscle & $\mathbf{\Delta}$ & $<$ & $=$ \\
\hline dystrophin & $=$ & $>$ & $>$ \\
\hline lamin, gamma 1 & $=$ & $>$ & $>$ \\
\hline bone morphogenetic protein 4 & $=$ & $>$ & $=$ \\
\hline collagen, type XIV, alpha 1 & $=$ & $>$ & $=$ \\
\hline connective tissue growth factor & $=$ & $>$ & $=$ \\
\hline fibromodulin & $=$ & $>$ & $=$ \\
\hline insulin-like growth factor 1 & $=$ & $>$ & $=$ \\
\hline integrin, beta 5 & $=$ & $>$ & $=$ \\
\hline integrin, alpha 10 & $=$ & $>$ & $=$ \\
\hline integrin, alpha 6 & $=$ & $>$ & $=$ \\
\hline osteonectin & $=$ & $>$ & $=$ \\
\hline thrombospondin 2 & $=$ & $>$ & $=$ \\
\hline integrin, alpha 11 & $=$ & $=$ & $>$ \\
\hline laminin, alpha 2 & $=$ & $=$ & $>$ \\
\hline sarcoglycan, delta & $=$ & $=$ & $>$ \\
\hline talin 1 & $=$ & $=$ & $>$ \\
\hline mothers against DPP homolog 6 & $=$ & $=$ & $<$ \\
\hline bone morphogenetic protein 7 & $=$ & $<$ & $=$ \\
\hline
\end{tabular}

Table 3. Summary of significant alterations in gene expression.

$\boldsymbol{\nabla}$ : decreased or $\boldsymbol{\Lambda}$ : increased gene expression after LVAD support, =: no change, $>$ :higher or <:lower expression pre- or post-LVAD compared to control. The shaded (green) genes are significantly altered in both DCM and IHD. 
Collagen $14 \alpha 1$ mRNA expression in DCM

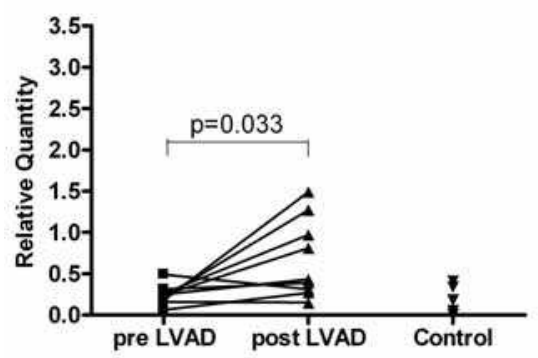

Decorin mRNA expression in DCM

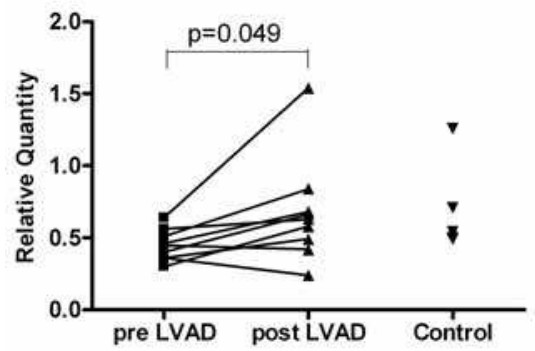

Collagen $14 \alpha 1$ mRNA expression in IHD

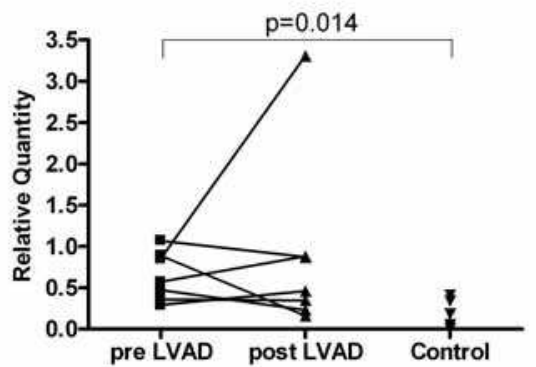

Decorin mRNA expression in IHD

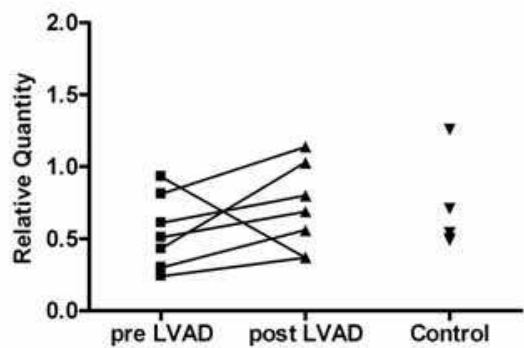

Fig. 4. Relative mRNA expression of genes encoding ECM proteins.

Relative mRNA expression was determined pre- and post-LVAD of DCM and IHD and tested in paired $t$-test. Increase of collagen 14a1 mRNA expression is significant in DCM but not in IHD. Compared to the control, only the mRNA expression pre-LVAD of IHD patients is significantly higher (unpaired t-test). Decorin is significantly increased post-LVAD in DCM. None of the pre- and post-LVAD samples differed significantly from the control samples.

\subsection{Genes encoding extracellular matrix proteins}

In DCM, 5 genes encoding ECM proteins were upregulated post-LVAD. However, except for collagen type VI alpha3, these genes did not differ significantly (either pre- or post LVAD) from control. This indicates that the increased expression of these 5 genes induced by the LVAD support is significant but as a group are not different from the control group (Figure 4). In IHD most differences between pre-LVAD and control were observed in genes encoding ECM proteins, but in post-LVAD samples these differences had disappeared, suggesting a high expression of ECM gene activity pre-LVAD (Figure 4).

Relative mRNA expression was determined pre- and post-LVAD of DCM and IHD and tested with the paired t-test. Increase of integrin a6 mRNA expression was significant in both DCM and IHD during LVAD support. Compared to the control, only the mRNA expression pre-LVAD of DCM patients is significantly lower (unpaired t-test). Integrin $\beta 6$ is 
only significantly increased post-LVAD in the IHD group. Compared to the control none of the pre- and post-LVAD samples differed significantly.

Integrin $\alpha 6$ mRNA expression in DCM

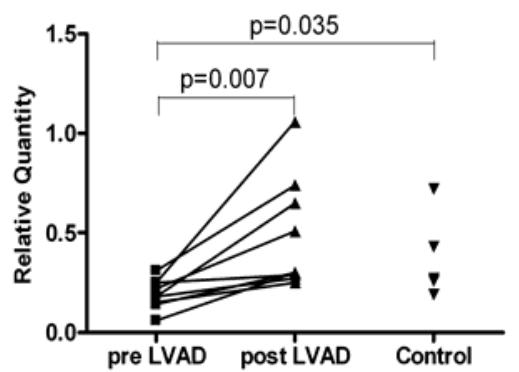

Integrin $\beta 6$ mRNA expression in DCM

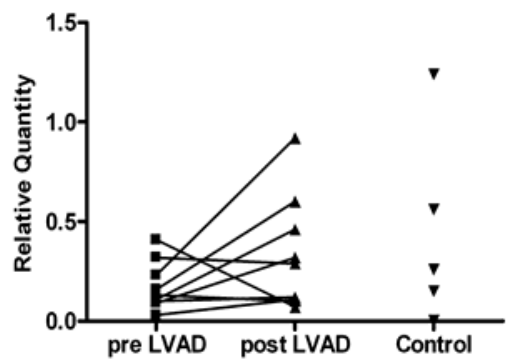

Integrin $\alpha 6$ mRNA expression in IHD

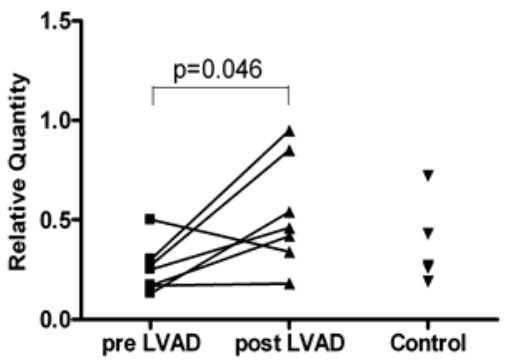

Integrin $\beta 6$ mRNA expression in IHD

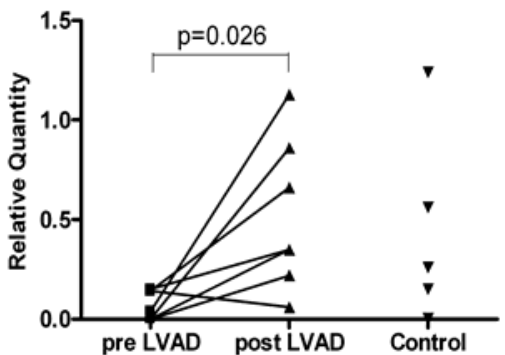

Fig. 5. Relative mRNA expression of genes encoding different integrins.

\subsection{Genes involved in the fibrotic pathway}

In the fibrotic pathway remarkable differences between DCM and IHD were observed. In DCM patients the expression of genes encoding pro-fibrotic factors (TGF $\beta 1$, FGF, IGF, endothelin and CTGF) remained unchanged, whereas the genes encoding anti-fibrotic proteins (BMP-4, BMP-7, decorin and ID1) increased after LVAD support. Pre-LVAD the expression of the pro-fibrotic factor FGF2 and the anti-fibrotic factor BMP-7 was low compared to control. Post-LVAD the expression of the anti-fibrotic factor BMP-4 was increased compared to control. However, in IHD patients these genes showed unchanged expression during LVAD support, but in pre-LVAD samples the pro-fibrotic genes are expressed stronger than in control and the anti-fibrotic gene BMP-7 is expressed less than in control. The post-LVAD expression pattern is comparable to that of control.

\subsection{Genes encoding basal membrane proteins}

The gene encoding osteopontin is the most remarkable member of the BM group. In both DCM and IHD patients, osteopontin expression is significantly reduced after LVAD 
support. Other BM proteins showed hardly any change, apart from laminin, vitronectin and thrombospondin (anchoring proteins).

Several integrins showed differential expression (mostly upregulation) in both DCM and IHD patients. In particular, integrin $\beta 6$ gene expression showed a strong increase after LVAD in the IHD group (Figure 5). Other membrane molecules, like caveolin, sarcoglycan and ATPase calcium transporting molecule, showed a low expression compared to control either pre- or post-LVAD.

\subsection{Genes encoding intracellular proteins}

Expression of some intracellular filament genes changed significantly after LVAD support in DCM (2/19: filamin, talin) and in IHD (1/19:desmoplakin), suggesting only a minor intracellular filament involvement. In this group it was remarkable that in IHD the gene encoding dystrophin was upregulated both pre- and post-LVAD.

Relatively many changes in the expression of signal transduction factors were observed after LVAD support both in DCM (2/8: Focal Adhesion Kinase and Ryanodine Receptor 2) and in IHD (4/8: catenin, myocyte enhancer factor 2A and 2C, and Ryanodine Receptor 2).

\section{Changes in miR expression during LVAD support}

Total RNA was isolated from heart tissue of heart failure patients pre- and post-LVAD. The relative quantities of miRNA1, miRNA133a, miRNA133b and miRNA-208 were established with the Taqman ${ }^{\circledR}$ MicroRNA assay (Applied Biosystems, Foster City, CA, USA). In Figure 6 the expression of miR-1, miR-133a and of miR133b is shown for DCM and IHD patients pre- and post-LVAD. Compared to control levels the miR expression in both heart failure groups was low for all miR tested. These low levels were more significant in IHD than in DCM. After LVAD support the levels did not change significantly, although in IHD there was a tendency that the miR expression levels return to normal. In patients with DCM we observed a tendency of further decrease. The expression of miR-208 showed similar changes (data not shown) as did the other three miRs. However, the expression was too low to make a reliable statistical analysis.

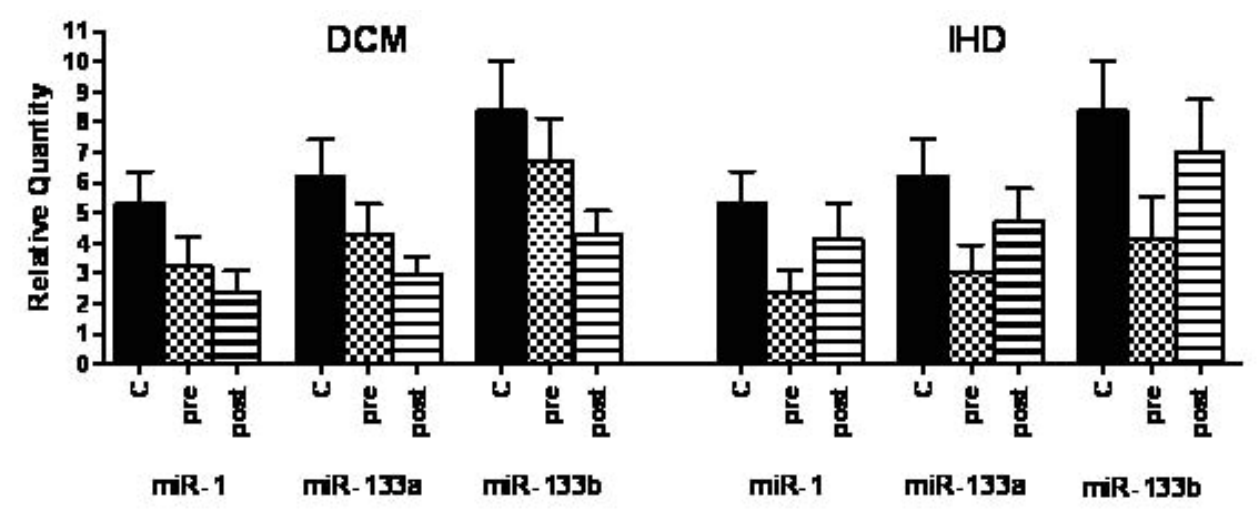

Fig. 6. Changes in miR expression after LVAD support 


\begin{tabular}{lcccccc}
\hline miR & \multicolumn{3}{c}{ DCM-patients } & \multicolumn{3}{c}{ IHD-patients } \\
\hline miR-1 & $\mathbf{1}$ & $\mathbf{2}$ & $\mathbf{3}$ & $\mathbf{1}$ & $\mathbf{2}$ & $\mathbf{3}$ \\
miR-133a & 0.20 & 0.24 & 0.06 & 0.08 & $\mathbf{0 . 0 5}$ & 0.44 \\
miR-133b & 0.73 & 0.19 & $\mathbf{0 . 0 4}$ & 0.11 & 0.07 & 0.35 \\
\hline
\end{tabular}

Table 4. Statistical analysis of miR expression changes after LVAD support.

The relative quantities of miR-1, miR-133a and miR-133b measured in heart tissue obtained from patients suffering from IHD $(n=8)$ or DCM $(n=9)$ or $C$ : controls $(n=5$; Pre = pre LVAD support ; Post $=$ post LVAD support.

The $\mathrm{p}$-values for the various differences in relative quantitative expression of the miRs in the myocardium obtained from DCM and IHD patients, respectively, before and after LVAD support. $\mathrm{P}<0.05$ is considered significant.

1: pre-LVAD versus post LVAD; 2: pre-LVAD versus control; 3: post LVAD versus control.

The results of the statistical analyses are presented in Table 4 . These data confirm that in DCM patients, LVAD support did not increase the low miR-expression. In patients with IHD the values of expression of the miRs after LVAD support were not significantly different from those in the controls, indicating that there was a tendency of the low levels of miRs to increase after LVAD in IHD patients.

\section{Discussion}

During unloading, the myocardium of the failing heart shows various changes, both macroscopically and microscopically. Major changes include reduction of cardiomyocyte size, and changes in the volumes of ECM and BM components (Goldsmith and Borg, 202; Bruggink et al., 2006; Parker and Ingber, 2007). In many studies analyzing the effect of mechanical support on heart failure, only marginal differences have been observed between IHD and DCM (Bruggink et al., 2006a; De Jonge et al., 2001, 2002; Grady et al., 2003). However, in the present study hierarchical clustering of all expressed genes in end-stage heart failure showed that DCM and IHD segregated and could be identified as separated entities (Figure 2). For this reason both groups were analyzed separately. In the IHD group pre- and post-LVAD samples did segregate by hierarchical clustering (Figure 3 ). In the DCM group no such separation of pre- and post-LVAD samples was observed. The explanation for this difference between both groups is unknown. DCM may have a genetic background that leads primarily to hypertrophy and fibrosis, leading to gene expression that differs from controls in several aspects, but is not completely reversed by LVAD support. By contrast, in IHD, the gene expression alterations that are induced by infarction are partly normalized by the unloading of the heart. The differences in mRNA expression between IHD and DCM may give an important clue in finding targets that are informative for the state of the (un)supported hearts.

In pre-LVAD samples of DCM patients, the expression of only 7 genes (2 up- and 5 downregulated) differed from control which increased to 10 genes post-LVAD ( 7 up- and 3 down-regulated). In IHD pre-LVAD samples, the expression of 15 genes (13 up- and 2 downregulated) differed from control, which decreased to 8 genes post-LVAD (7 up- and only 1 down-regulated). In both groups most genes that were differentially expressed pre-LVAD normalized to control levels after LVAD support. On the other hand, LVAD support can also induce a down- or upregulation of genes of which the pre-LVAD levels did not differ from 
control level (Table 2). Eleven genes showed significant changes pre- and post-LVAD in both DCM and IHD. However, only 3 genes showed changes that were the same in DCM and IHD (calcium channel alpha $1 \mathrm{C}$ subunit, integrin-a11 and ryanodine receptor 2).

In DCM, the expression of caveolin remained low (both pre- and post-LVAD) compared to control. This is in contrast to the described up-regulation of caveolin protein after LVAD support (Uray et al., 2003). In IHD the expression of dystrophin and laminin (gamma 1) remained high after LVAD support. Changes in expression of both genes after LVAD have been described by others (Vatta et al., 2004; Birks et al., 2005; Refaat et al., 2008).

LVAD-induced changes in ECM and cardiomyocytes have been described by others as well (Milting et al., 2008; Bruggink et al 2006b; Thohan et al., 2005). In this respect, the total number of genes coding for various structural elements, that were differentially expressed pre- and post-LVAD was surprisingly low. Morphological changes during LVAD support were paralleled by changes in collagen turnover and expression of genes encoding for structural collagens (Type I and III; Bruggink et al, 2006b; 2007). So, the minor changes observed in expression of ECM genes in the present study may imply that most ECM changes are induced post-transcriptionally, either by micro-RNA regulation (Schipper et al., 2008) or in the matrix itself (e.g. by MMP). The latter is supported by significant changes in mRNA expression of MMP during LVAD support (Li et al., 2001; Klotz et al., 2005). Interestingly the anchoring and connecting collagens (types VI, XIV and XV) and molecules involved in ECM assembly like fibulin, fibronectin, osteonectin and proteoglycans (fibromodulin, heparan sulfate and decorin; Pollard et al., 2008) changed upon LVAD, although not similar in DCM and IHD patients. The LVAD-induced changes in the expression of these molecules, including collagen, also observed by others (Jahanyar et al., 2007; Gabrielsen et al., 2007), may contribute to the increased rigidity of the heart after LVAD support (Klotz et al., 2005).

Previously, we have shown that unloading of the left ventricle decreased the immunohistochemical expression of collagen IV in the BM (Bruggink et al., 2007). In contrast, immunoreactivity of laminin did not show substantial changes upon LVAD. Of the 17 tested genes that encode BM proteins only few showed expression changes after LVAD, indicating a dysbalance between mRNA expression and protein expression. The few genes that did show changes upon LVAD, either in DCM or IHD, are involved in cell-adhesion (laminin $\beta 1$ and $\gamma 1$, osteopontin). Together with the changes observed in the gene expression of the integrin, cadherin and sarcoglycan family members, these results underline the importance of these specific anchoring or connecting proteins in the structural changes observed (Birks et al.,2005; Gabrielsen et al., 2007; Latif et al., 2007; Kim et al.,1999).

Only minor changes were observed in the expression of genes encoding intracellular cytoskeleton proteins. In DCM, alterations after LVAD support in cytoskeletal filaments (dystrobrevin, filamin, junction plakoglobin, and talin) are more pronounced than in IHD (desmoplakin, dystrophin and talin; Gabrielsen et al., 2007). This could indicate that this class of genes is more affected in DCM than in IHD, which may be explained by the different onset of myocardial damage in both diseases.

In the fibrotic pathway a remarkable difference between DCM and IHD is observed. In DCM the expression of pro-fibrotic factors (TGF $\beta 1$, FGF, IGF, endothelin and CTGF) did not change upon LVAD support, but the expression of anti-fibrotic genes (BMP-4, BMP-7, decorin, and Id1) increased. This is paralleled by reduced fibrosis in DCM (Bruggink et al., 2006b). In patients with IHD the expression of both anti- and pro-fibrotic factors remain unchanged upon LVAD support. However, in IHD the pre-LVAD expression levels of the 
pro-fibrotic response genes are stronger than in control whereas the expression of the antifibrotic gene BMP-7 is lower than in control. This will favour fibrosis in the hearts of patients with IHD. In these patients, the post-LVAD situation may be associated with a return of gene expression to control values. This may lead to a reduction of fibrosis as is shown in various studies. So, pro- and anti-fibrotic gene expression is in agreement with previously described reduction of fibrosis after LVAD support (Goldsmith and Borg, 2002; Gabrielsen et al., 2007), although the mechanisms responsible differed between the two entities.

In view of the changes in mRNA expression that did not seem to be paralleled by corresponding protein expression, special emphasis was given to miR expression during LVAD support. These miRs are important in the post-transcriptional regulation of mRNAs, also in the heart (Chen, 2007; Couzin, 2008). The miRs tested (miR-1, miR-133a and miR133b) had relatively low expression in the myocardium of heart failure patients compared to controls. In IHD patients the level of miR expression tended to return to control levels upon LVAD support. In DCM, however, the miR expression levels tended to decrease even further, which suggests that genes under the control of these miRs could be expressed even stronger. Chen et al. (2006) have described that miR-1 and miR-133 promote skeletal muscle myogenesis and myoblast proliferation, respectively (Townley-Tilson et al, 2010). Similar data have been produced by Liu et al. (2007) and Ikeda et al. (2008) for the failing myocardium. The relatively low expression of miRs in the failing heart, compared to control, may be related to the presence of myocardial hypertrophy (De Jonge et al., 2002), as overexpression of both miR-1 and miR-133 leads to cardiac hypertrophy (Care et al., 2007). This difference in miR expression between DCM and IHD patients after LVAD support may be explained by the lack of need for cell proliferation in DCM unlike in IHD where there is a need for cell proliferation. Remodeling of DCM involves mainly a reduction of hypertrophy of cardiomyocytes, whereas IHD involves tissue repair including cell proliferation. This may indicate that the studied miRs are primarily involved in regulation of proliferative processes rather than in reduction of hypertrophy. As already mentioned, the reduction in miRNA expression in IHD patients is not restored completely to control levels during LVAD support, not in patients supported for a short period of time nor in patients supported for more than over one year.

The miR data do show that myocardial expression of miRs changes upon heart failure (Busk and Cirera, 2010) and upon LVAD support. In that respect it is interesting to note that there are initial indications that miR released in the serum (Cheng et al. 2010) may act as biomarkers to screen for cardiac diseases (Adachi et al., 2010) and be targets for therapy (Seok and Wang, 2010).

In conclusion, the set of genes coding for proteins involved in mechanotransduction, selected for the analysis of changes in mRNA expression pre- and post-LVAD, resulted in an identification of IHD and DCM as separate entities. The morphologic and structural changes observed in the failing human heart upon LVAD support are only partly reflected in changes of mRNA expression of genes encoding proteins involved in mechanotransduction. This suggests that most changes in ECM and intracellular filaments are not regulated at the mRNA level. However, expression of genes encoding membrane-bound proteins such as cadherin and integrins, and anchoring proteins such as collagen type VI and proteoglycans, is clearly affected by LVAD support and contributes to adaptation to improved loading conditions. Also the genes involved in fibrosis showed adaptation to LVAD support, and their expression runs parallel to the observed morphological changes. These genes may 
prove to be important biomarkers in the development of protocols which decide whether LVAD supported patients should undergo heart transplantation, can be weaned from the device, or could rather continue their LVAD therapy for a longer period of time. The role of miR as biomarkers in this decision making, but also as therapeutic targets, is promising but still needs further investigation (Montgomery and van Rooij, 2010).

\section{Acknowledgement}

MFM van Oosterhout was supported by a grant from the Dutch Heart Foundation; project number 2004T31.

\section{References}

Adachi , T.; Nakanishi, M.; Otsuka, Y.; Nishimura, K.; Hirokawa, G.; Goto, Y.; Nonogi, H. \& Iwai, N. (2010). Plasma microRNA 499 as a biomarker of acute myocardial infarction. Clin Chem 56(7), 1183-1185.

Birks, E.J.; Hall, J.L.; Barton, P.J.R.; Grindle, S.; Latif, N.; Hardy, J.P.; Rider, J.E.; Banner, N.R.; Khaghani, A.; Miller, L.W. \& Yacoub, M.H. (2005). Gene profiling changes in cytoskeletal proteins during clinical recovery after left ventricular-assist device support. Circulation 112(9), 157-164.

Brower, G.L.; Gardner, J.D.; Forman, M.F.; Murray, D.B.; Voloshenyuk, T.; Levick, S.P.;.Janicki, J.S. (2006) The relationship between myocardial extracellular matrix remodeling and ventricular function. Eur JCardiothorac Surg 30(4), 604-610.

Bruggink, A.H.; de Jonge, N.; van Oosterhout, M.F.; Van Wichen, D.F.; de Koning, E.; Lahpor, J.R.; Kemperman, H.; Gmelig-Meyling, F.H.J. \& de Weger, R.A. (2006a). Brain natriuretic peptide is produced both by cardiomyocytes and cells infiltrating the heart in patients with severe heart failure supported by a left ventricular assist device. JHeart Lung Transplant 25(2), 174-80.

Bruggink, A.H.; van Oosterhout, M.F.; de Jonge, N.; Ivangh, B.; Van Kuik, J.; Voorbij, R.H.A.M.; Cleutjens, J.P.M.; Gmelig-Meyling, F.H.J. \& de Weger, R.A. (2006b). Reverse remodeling of the myocardial extracellular matrix after prolonged left ventricular assist device support follows a biphasic pattern. JHeart Lung Transplant 25(9), 1091-1098.

Bruggink, A.H.; van Oosterhout, M.F.M.; de Jonge, N.; Cleutjens, J.P.M.; Van Wichen, D.F.; van Kuik, J.; Tilanus, M.G.J.; Gmelig-Meyling, F.H.J.; van den Tweel, J.G.; De Weger, R.A. (2007). Type IV collagen degradation in the myocardial basement membrane after unloading of the failing heart by a left ventricular assist device. $L a b$ Invest 87(11), 1125-1137.

Bruggink, A.H.; van Oosterhout, M.F.; De Jonge, N.; Gmelig-Meyling, F.H.; De Weger, R.A. (2008). TNF alpha in patients with end-stage heart failure on medical therapy or supported by a left ventricular assist device. Transpl Immunol 19(1), 64-68.

Busk, P.K. \& Cirera, S. (2010). MicroRNA profiling in early hypertrophic growth of the left ventricle in rats. Biochem Biophys Res Commun 396(4), 989-993.

Carè, A.; Catalucci, D.; Felicetti, F.; Bonci, D.; Addario, A.; Gallo, P.; Bang, M.-L.; Segnalini, P.; Gu Y.S.; Dalton, N.D.; Elia, L.; Latronico, M.V.G.; Hoydal, M.; Autore, C.; Russo, M.A.; Dorn, G.W.; Ellingsen, O.; Ruiz-Lozano, P.; Peterson, K.L.; Croce, C.M.; 
Peschle, C. \& Condorelli, G. (2007). MicroRNA-133 controls cardiac hypertrophy. Nat Med 13(5), 613-618.

Chen, J.F.; Mandel, E.M.; Thomson, J.M.; Wu, Q.L.; Callis, T.E.; Hammond, S.M.; Conlon, F.L. \& Wang, D.Z. (2006). The role of microRNA-133 in skeletal muscle proliferation and differentiation. Nat Genet 38(2), 228-233.

Chen, K.R. (2007). MicroRNAs and the tell-tale heart. Nature 447(7144), 389-922.

Cheng, Y.; Ji, R.; Yue, J.; Yang, J.; Liu, X.; Chen, H.; Dean, D.B. \& Zhang, C. (2007). MicroRNAs Are Aberrantly Expressed in Hypertrophic Heart: Do They Play a Role in Cardiac Hypertrophy? Am JPathol 170 (4), 1831-1840.

Cheng, Y.H.; Tan, N.; Yang, J.A.; Liu, X.J.; Cao, X.P.; He, P.C.; Dong, X.L.; Qin, S.S \& Zang, C.X. (2010). A translational study of circulating cell-free miRNA-1 in acuite myocardial infarction. Clin Sc 119 (1-2), 87-95.

Coutinho, L.L.; Matukumalli, L.K.; Sonstergard, T.S.; Van Tassell, C.P.; Gasbarre, L.C.; Capuco, A.V. \& Smith, T.P.L. (2007). Discovery and profiling of bovine microRNAs from immune-related and embryonic tissues. Physiol Genomics 29(1), 35-43.

Couzin, J. (2008). MicroRNAs make big impression in disease after disease. Science 319(5871), 1782-1784.

De Jonge, N.; Kirkels, H.; Lahpor, J.R.; Klopping, C.; Hulzebos, E.J.; de la Riviere, A.B. \& de Medina, E.O.R. (2001). Exercise performance in patients with end stage heart failure after implantation of a left ventricular assist device and after heart transplantation: an outlook for permanent assisting? JAm Coll Cardiol 37(7), 1794-1799.

De Jonge, N.; Van Wichen, D.F.; Schipper, M.E.; Lahpor, J.R.; Gmelig-Meyling, F.H.J.; de Medina, E.O. \& De Weger, R.A. (2002). Left ventricular assist device in end-stage heart failure: persistence of structural myocyte damage after unloading. An immunohistochemical analysis of the contractile myofilaments. JAm Coll Cardiol 39(6),963-969.

De Weger, R. \& De Jonge, N. (2009). Editorial comment heart transplantation. Curr Opin Organ Transplant 14(5), 552-553.

Estrada-Quintero, T.; Uretsky, B.F.; Murali, S.; Griffith, B.P. \& Kormos, R.L. (1995). Neurohormonal activation and exercise function in patients with severe heart failure and patients with left ventricular assist system. A comparative study. Chest 107(6), 1499-1503.

Frazier, O.H. \& Myers, T.J. (1999). Left ventricular assist system as a bridge to myocardial recovery. Ann Thorac Surg 68(2), 734-41.

Gabrielsen, A. ; Lawler, P.R. ; Wang, Y.Z. ; Steinbruchel, D.; Blagoja, D.; Paulsson-Berne, G.; Kastrup, J. \& Hansson, G.K. (2007). Gene expression signals involved in ischemic injury, extracellular matrix composition and fibrosis defined by global mRNA profiling of the human left ventricular myocardium. JMol Cell Cardiol 42(4), 870883.

Goldsmith, E.C. \& Borg, T.K. (2002). The dynamic interaction of the extracellular matrix in cardiac remodeling. JCard Fail 8(6), S314-S318.

Grady, K.L.; Meyer, P.M.; Mattea, A.; Dressler, D.; Ormaza, S.; White-Williams, C.; Chillcott, S.; Kaan, A.; Loo, A.; Todd, B.; Klemme, A.; Piccione, W. \& Costanzo, M.R. (2003). Change in quality of life from before to after discharge following left ventricular assist device implantation. JHeart Lung Transplant 22(3) 322-333. 
Ikeda, S.; Kong, S.W.; Lu, J.; Bisping, E.; Zhang, H.; Allen, P.D.; Golub, T.R.; Pieske, B. \& Pu, W.T. (2007). Altered microRNA expression in human heart disease. Physiol Genom 31(3), 367-373.

Jahanyar, J. ; Joyce, D.L. ; Southard, R.E. ; Loebe, M.; Noon, G.P.; Koerner, M.M.; TorreAmione, G.\& Youker, K.A. (2007). Decorin-mediated transforming growth factorbeta inhibition ameliorates adverse cardiac remodeling. J Heart Lung Transplant 26(1), 34-40.

Ji, R.R.; Cheng,Y.H.; Yue, J.M.; Yang, J.; Liu, X.; Chen, H.; Dean, D.B. \& Zhang, C.X. (2007). croRNA expression signature and antisense-mediated depletion reveal an essential role for microRNA in vascular neointimal lesion formation. Circ Res 100(11), 15791588.

Kemperman, H.; van den Berg, M.; Kirkels, H. \& De Jonge, N. (2004). B-type natriuretic peptide (BNP) and N-terminal proBNP in patients with end-stage heart failure supported by a left ventricular assist device. Clin Chem 50(9), 1670-1672.

Kim, H.; Yoon, C.S. \& Rah, B. (1999). Expression of extracellular matrix components fibronectin and laminin in the human fetal heart. Cell Struct Funct 24(1), 19-26.

Klotz, S.; Foronjy, R.F.; Dickstein, M.L.;Garrelds, I.M.; Danser, A.H.J.; Oz, M.C.; D'Armiento, J. \& Burkhoff, D. (2005). Mechanical unloading during left ventricular assist device support increases left ventricular collagen cross-linking and myocardial stiffness. Circulation 112(3), 364-374.

Latif, N.; Yacoub, M.H.; George, R.; Barton, P.J. \& Birks EJ. (2007). Changes in sarcomeric and non-sarcomeric cytoskeletal proteins and focal adhesion molecules during clinical myocardial recovery after left ventricular assist device support. JHeart Lung Transplant 26(3), 230-235.

Li, Y.Y. ; Feng, Y.Q.; McTiernan, C.F. ; Moravec, C.S.; Wang, P.; Rosenblum, W.; Kormos, R.L. \& Feldman, A.M. (2001). Downregulation of matrix metalloproteinases and reduction in collagen damage in the failing human heart after support with left ventricular assist devices. Circulation 104(10), 1147-1152.

Liu, N.; Williams, A.H.; Kim, Y.; McAnally, J.; Bezprozvannaya, S.; Sutherland, L.B.; Richardson, J.A.; Bassel-Duby, R. \& Olson, E.N. (2007). An intragenic MEF-2 dependent enhancer directs muscle-specific expression of microRNAs 1 and 133. Proc Nat Acad Sci USA 104(52), 20844-20849.

Markham, D.W. \& Hill, J.A. (2010). MicroRNAs and heart failure diagnosis: MiR-acle or MiR-age?. Circ Res 106 (4), 1011-1013.

Milting, H.; Ellinghaus, P.; Seewald, M.; Cakar, H.; Bohms, B.; Kassner, A.; Koerfer, R.; Klein, M.; Krahn, T.; Kruska, L.; El Banayosy, A.. \& Kramer, F. (2008). Plasma biomarkers of myocardial fibrosis and remodeling in terminal heart failure patients supported by mechanical circulatory support devices. JHeart Lung Transplant 27(6), 589-596.

Montgomery, R.L. \& Van Rooij, E (2010). MicroRNA regulation as a therapeutic strategy for cardiovascular disease. Curr Drug Targets 11 (8), 936-942.

Muller, J.; Wallukat, G.; Weng, Y.G.; Dandel, M.; Spiegelsberger, S.; Semrau, S.; Brandes, K.; Theodoridis, V.; Loebe, M.; Meyer, R. \& Hetzer, R. (1997). Weaning from mechanical cardiac support in patients with idiopathic dilated cardiomyopathy. Circulation 96(2), 542-549. 
Parker, K.K. \& Ingber, D.E. (2007). Extracellular matrix, mechanotransduction and structural hierarchies in heart tissue engineering. Philos Trans R Soc Lond B Biol Sci. 362(1484), 1267-1279.

Pollard, T.D. \& Earnshaw, W.C. (2008). Section VIII Cellular adhesion and the extracellular matrix. In: Cell Biology, Philadelphia, Saunders Elsevier, pp 513-598.

Refaat, M.; Chemaly, E.; Lebeche, D.; Gwathmey, J.K. \& Hajjar, R.J. (2008). Ventricular arrhythmias after left ventricular assist device implantation. Pacing Clin Electrophysiol 31(10), 1246-1252.

Schipper, M.E.; van Kuik, J.; de Jonge, N.; Dullens, H.F. \& De Weger, R.A. (2008). Changes in regulatory microRNA expression in myocardium of heart failure patients on left ventricular assist device support. JHeart Lung Transplant 27(12), 1282-1285.

Schuldt, A. (2010). Great expectations of small RNAs. Nature Revs Mol Cell Biol 11, 27.

Seok, H.Y. \& Wang, D.Z. (2010). The emerging role of microRNAs as a therapeutic target for cardiovascular disease. Biodrugs 24(3), 147-155.

Spinale, F.G. (2002). Matrix metalloproteinases: regulation and dysregulation in the failing heart. Circ Res 90(4), 520-530.

Stamenkovic, I. (2003). Extracellular matrix remodelling: the role of matrix metalloproteinases. JPathol 200(4), 448-464.

Thohan, V.; Stetson, S.J.; Nagueh, S.F.; Rivas-Gotz, C.; Koerner, M.M.; Lafuente, J.A.; Loebe, M.; Noon, G.P. \& Torre-Amione, G. (2005). Cellular and hemodynamics responses of failing myocardium to continuous flow mechanical circulatory support using the DeBakey-Noon left ventricular assist device: A comparative analysis with pulsatiletype devices. JHeart Lung Transplant 24(5), 566-575.

Townley-Tilson, W.H.D.; Callis, T.E. \& Wang, D.Z. (2010). MicroRNAs 1, 133, and 206: Critical factors of skeletal and cardiac muscle development, function and disease. Int. . I Biochem. Cell Biol 42 (8), 1252-1255.

Uray, I.P.; Connelly, J.H. \& Frazier, O.H.; Taegtmeyer, H. \& Davies, P.J. (2003). Mechanical unloading increases caveolin expression in the failing human heart. Cardiovasc Res 59(1), 57-66.

Van Rooij, E.; Sutherland, L.B.; Liu, N.; Williams, A.H.; McAnally, J.; Gerard, R.D.; Richardson, J.A. \& Olson, E.N. (2006). A signature pattern of stress responsive microRNAs that can evoke cardiac hypertrophy and heart failure. Proc Natl Acad Sci USA 103(48), 18255-60.

Vatta, M,; Stetson, S.J.; Jimenez, S.; Entman, M.L.; Noon, G.P.; Bowles, N.E.; Towbin, J.A. \& Torre-Amione, G. (2004). Molecular normalization of dystrophin in the failing left and right ventricle of patients treated with either pulsatile or continuous flow-type ventricular assist devices . JAm Coll Cardiol 43(3), 811-817. 


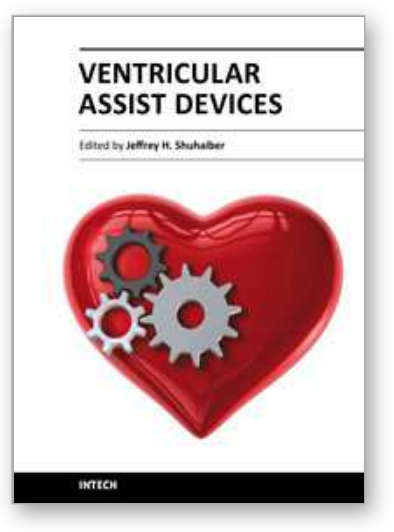

\author{
Ventricular Assist Devices \\ Edited by Dr. jeffrey Shuhaiber
}

ISBN 978-953-307-164-0

Hard cover, 212 pages

Publisher InTech

Published online 26, April, 2011

Published in print edition April, 2011

The assist devices will continue adding a large number of years of life to humans globally and empower the medical society to optimize heart failure therapy. While expensive and cumbersome task, the foundation provided in this book reflects a contemporary product of original research from a multitude of different experts in the field. We hope this cumulative international effort provides the necessary tools for both the novice as well as the active practitioner aiming to change the outcome of these complex patients.

\title{
How to reference
}

In order to correctly reference this scholarly work, feel free to copy and paste the following:

Marguérite E.I. Schipper, Sjoukje I. Lok, Hub Dullens, Joyce Van Kuik, Frits H.J. Gmelig-Meyling, Jaap Lahpor, Marc A. Vos, Arnoud Van Der Laarse, Nicolaas De Jonge, Matthijs F.M. Van Oosterhout and Roel A. De Weger (2011). Altered Expression of mRNA and miRNA during Mechanical Support of the Failing Human Heart, Ventricular Assist Devices, Dr. jeffrey Shuhaiber (Ed.), ISBN: 978-953-307-164-0, InTech, Available from: http://www.intechopen.com/books/ventricular-assist-devices/altered-expression-of-mrna-and-mirnaduring-mechanical-support-of-the-failing-human-heart

\section{INTECH}

open science | open minds

\section{InTech Europe}

University Campus STeP Ri

Slavka Krautzeka 83/A

51000 Rijeka, Croatia

Phone: +385 (51) 770447

Fax: +385 (51) 686166

www.intechopen.com

\section{InTech China}

Unit 405, Office Block, Hotel Equatorial Shanghai

No.65, Yan An Road (West), Shanghai, 200040, China

中国上海市延安西路65号上海国际贵都大饭店办公楼 405 单元

Phone: +86-21-62489820

Fax: $+86-21-62489821$ 
(C) 2011 The Author(s). Licensee IntechOpen. This chapter is distributed under the terms of the Creative Commons Attribution-NonCommercialShareAlike-3.0 License, which permits use, distribution and reproduction for non-commercial purposes, provided the original is properly cited and derivative works building on this content are distributed under the same license. 